\title{
Physical Quantity to Physical Quantity Ratio Data Type
}

National Cancer Institute

\section{Source}

National Cancer Institute. Physical Quantity to Physical Quantity Ratio Data Type. NCI

Thesaurus. Code C95676.

A data type comprised of a physical quantity divided by another physical quantity. 